Article

\title{
New Multigranulation Neutrosophic Rough Set with Applications
}

\author{
Chunxin Bo ${ }^{1}$, Xiaohong Zhang ${ }^{2, *} \mathbb{C}$, Songtao Shao ${ }^{1} \mathbb{C}$ and Florentin Smarandache ${ }^{3} \mathbb{C}$ \\ 1 College of Information Engineering, Shanghai Maritime University, Shanghai 201306, China; \\ 201640311001@stu.shmtu.edu.cn (C.B.); 201740310005@stu.shmtu.edu.cn (S.S.) \\ 2 Department of Mathematics, Shaanxi University of Science \& Technology, Xi'an 710021, China \\ 3 Department of Mathematics, University of New Mexico, Gallup, NM 87301, USA; smarand@unm.edu \\ * Correspondence: zhangxiaohong@sust.edu.cn or zhangxh@shmtu.edu.cn
}

Received: 3 September 2018; Accepted: 6 October 2018; Published: 2 November 2018

\begin{abstract}
After the neutrosophic set (NS) was proposed, NS was used in many uncertainty problems. The single-valued neutrosophic set (SVNS) is a special case of NS that can be used to solve real-word problems. This paper mainly studies multigranulation neutrosophic rough sets (MNRSs) and their applications in multi-attribute group decision-making. Firstly, the existing definition of neutrosophic rough set (we call it type-I neutrosophic rough set $\left(\mathrm{NRS}_{\mathrm{I}}\right)$ in this paper) is analyzed, and then the definition of type-II neutrosophic rough set $\left(\mathrm{NRS}_{\mathrm{II}}\right)$, which is similar to $\mathrm{NRS}_{\mathrm{I}}$, is given and its properties are studied. Secondly, a type-III neutrosophic rough set $\left(\mathrm{NRS}_{\text {III }}\right)$ is proposed and its differences from NRS I and NRS ${ }_{\text {II }}$ are provided. Thirdly, single granulation NRSs are extended to multigranulation NRSs, and the type-I multigranulation neutrosophic rough set $\left(\mathrm{MNRS}_{\mathrm{I}}\right)$ is studied. The type-II multigranulation neutrosophic rough set $\left(\mathrm{MNRS}_{\mathrm{II}}\right)$ and type-III multigranulation neutrosophic rough set $\left(\mathrm{MNRS}_{\mathrm{III}}\right)$ are proposed and their different properties are outlined. We found that the three kinds of MNRSs generate tcorresponding NRSs when all the NRs are the same. Finally,

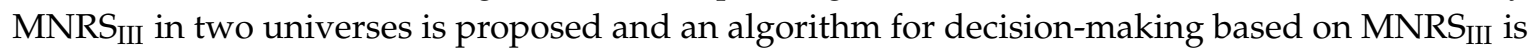
provided. A car ranking example is studied to explain the application of the proposed model.
\end{abstract}

Keywords: inclusion relation; neutrosophic rough set; multi-attribute group decision-making (MAGDM); multigranulation neutrosophic rough set (MNRS); two universes

\section{Introduction}

Many theories have been applied to solve problems with imprecision and uncertainty. Fuzzy set (FS) theories [1-3] use the degree of membership to solve the fuzziness. Rough set (RS) theories [4-7] deal with uncertainty by lower and upper approximation (LUA). Soft set theories [8-10] deal with uncertainty by using a parametrized set. However, all these theories have their own restrictions. Smarandache proposed the concept of the neutrosophic set (NS) [11], which was a generalization of the intuitionistic fuzzy set (IFS). To address real-world uncertainty problems, Wang et al. proposed the single-valued neutrosophic set (SVNS) [12]. Many theories about neutrosophic sets were studied and extended single-valued neutrosophic set [13-15]. Zhang et al. [16] analyzed two kinds of inclusion relations of the NS and then proposed the type-3 inclusion relation of NS. The combinations of the FS and RS are popular and produce many interesting results [17]. Broumi and Smarandache [18] combined the RS and NS, then produced a rough NS and studied its qualities. Yang et al. [19] combined the SVNS and RS, then produced the SVNRS (single-valued neutrosophic rough set) and studied its qualities.

From the view point of granular computing, the RS uses upper and lower approximations to solve uncertainty problems, shown by single granularity. However, with the complexity of 
real-word problems, we often encounter multiple relationship concepts. Qian and Liang [20] proposed a multigranularity rough set (MGRS). Many scholars have generalized MGRS and acquired some interesting consequences [21-26]. Zhang et al. [27] proposed non-dual MGRSs and investigated their qualities.

Few articles have been published about the combination of NSs and multigranulation rough sets. In this paper, we study three kinds of neutrosophic rough sets (NRSs) and multigranulation neutrosophic rough sets (MNRSs) that are based on three kinds of inclusion relationships of NS and corresponding union and intersection relationships $[11,12,16]$. Their different properties are discussed. We found that MNRSs degenerate to corresponding NRSs when the NRs are the same. Yang et al. [19] defined the NRS ${ }_{I}$ and considered its properties. Bo et al. [28] proposed $\mathrm{MNRS}_{I}$ and discussed its properties. In this paper, we study $\mathrm{NRS}_{\text {II }}$ and $\mathrm{MNRS}_{\text {II }}$. We also study $\mathrm{NRS}_{\text {III }}$ and MNRS $_{\text {III }}$, which are based on a type-3 inclusion relationship and corresponding union and intersection relationships. Finally, we use MNRS III on two universes to solve multi-attribute group decision-making (MAGDM) problems.

The structure of this article is as follows: In Section 2, some basic notions and operations of NRS I and NRS II are introduced. In Section 3, the definition of $\mathrm{NRS}_{\text {III }}$ is proposed and its qualities are investigated, and the differences between $\mathrm{NRS}_{\mathrm{I}}, \mathrm{NRS}_{\mathrm{II}}$, and $\mathrm{NRS}_{\text {III }}$ are illustrated using an example. In Section 4, MNRS I and MNRS II are discussed. In Section 5, MNRS III is proposed and its differences from MNRS I and MNRS II are studied. In Section 6, MNRS III on two universes is proposed and an application to solve the MAGDM problem is outlined. Finally, Section 7 provides our conclusions and outlook.

\section{Preliminary}

In this chapter, we look back at several basic concepts of type-I NRS, then propose the definition and properties of type-II NRS.

Definition 1. [12] A single valued neutrosophic set A in X is denoted by:

$$
A=\left\{\left(x, T_{A}(x), I_{A}(x), F_{A}(x)\right) \mid x \in X\right\},
$$

where $T A(x), I A(x), F A(x) \in[0,1]$ for each point $x$ in $X$ and satisfies the condition $0 \leq T_{A}(x)+I_{A}(x)+F_{A}(x) \leq 3$.

For convenience, "SVNS" is abbreviated to "NS" later. Here, NS(X) denotes the set of all SVNS in X.

Definition 2. [29] A neutrosophic relation (NR) is a neutrosophic fuzzy subset of $X \times Y$, that is, $\forall x \in X$, $y \in Y$,

$$
R(x, y)=\left(T_{R}, I_{R}, F_{R}\right),
$$

where $T_{R}: X \times Y \rightarrow[0,1], I_{R}: X \times Y \rightarrow[0,1]$, and $F_{R}: X \times Y \rightarrow[0,1]$ and satisfies $0 \leq T_{R}+I_{R}+F_{R} \leq 3$. $N R(X \times Y)$ denotes all the $N R s$ in $X \times Y$.

Definition 3. [19] Suppose $(U, R)$ is a neutrosophic approximation space (NAS). $\forall A \in N S(U)$, the LUA of $A$, denoted by $\underline{R}(A)$ and $\bar{R}(A)$, is defined as: $\forall x \in U$,

$$
\underline{R}(A)=\underset{y \in U}{\cap}\left(R^{\mathcal{C}}(x, y) \cup A(y)\right), \bar{R}(A)=\underset{y \in U}{\cup}(R(x, y) \cap A(y)) .
$$

The pair $(\underline{R}(A), \bar{R}(A))$ is called the SVNRS of $A$. In this paper, we called it type-I neutrosophic rough set $\left(\mathrm{NRS}_{\mathrm{I}}\right)$. Because the definition of $\mathrm{NRS}_{\mathrm{I}}$ is based on the type-1 operator of NS, the definition can be written as:

$$
\underline{\operatorname{NRS}_{I}}(A)=\underset{y \in U}{\cap_{1}}\left(R^{c}(x, y) \cup_{1} A(y)\right), \overline{N R S_{I}}(A)=\underset{y \in U}{\cup_{1}}\left(R(x, y) \cap_{1} A(y)\right) .
$$


Proposition 1. [19] Suppose $(U, R)$ is an NAS. $\forall A, B \in N S(U)$, we have:

(1) If $A \subseteq_{1} B$, then $\operatorname{NRS}_{I}(A) \subseteq_{1} \operatorname{NRS}_{I}(B)$ and $\overline{N R S_{I}}(A) \subseteq_{1} \overline{N R S_{I}}(B)$.

(2) $N R S_{I}\left(A \cap_{1} B\right)=N R S_{I}(A) \cap_{1} N R S_{I}(B), \overline{N R S_{I}}\left(A \cup_{1} B\right)=\overline{N R S_{I}}(A) \cup_{1} \overline{N R S_{I}}(B)$.

(3) $\overline{N R S_{I}}(A) \cup_{1} \underline{N R S_{I}(B) \subseteq} \subseteq_{1} \underline{N R S_{I}\left(A \cup_{1} B\right)}, \overline{N R S_{I}}\left(A \cap_{1} B\right) \subseteq_{1} \overline{N R S_{I}}(A) \cap_{1} \overline{N R S_{I}}(B)$.

According to the $\mathrm{NRS}_{\mathrm{I}}$, we can get the definition and properties of $\mathrm{NRS}_{\text {II }}$, which is based on the type-2 operator of NS.

Definition 4. Suppose $(U, R)$ is an NAS. $\forall A \in N S(U)$, the type-II LUA of $A$, is defined as:

$$
\underline{N R S_{I I}}(A)=\underset{y \in U}{\cap_{2}}\left(R^{c}(x, y) \cup_{2} A(y)\right), \overline{N R S_{I I}}(A)=\underset{y \in U}{\cup_{2}}\left(R(x, y) \cap_{2} A(y)\right)
$$

The pair $\left(\underline{N R S_{I I}}(A), \overline{N R S_{I I}}(A)\right)$ is called $\mathrm{NRS}_{\text {II }}$ of $A$.

Proposition 2. Suppose $(U, R)$ is an NAS. $\forall A, B \in N S(U)$, we have:

(1) If $A \subseteq_{2} B$, then $N R S_{I I}(A) \subseteq_{2} \operatorname{NRS}_{I I}(B), \overline{N R S_{I I}}(A) \subseteq_{2} \overline{N R S_{I I}}(B)$.

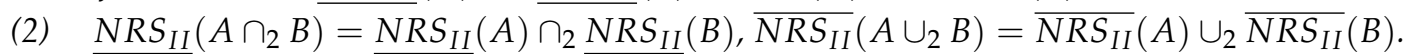

(3) $\overline{N R S_{I I}}(A) \cup_{2} \underline{N R S_{I I}(B) \subseteq_{2}} \underline{N R S_{I I}\left(A \cup \cup_{2} B\right)}, \overline{N R S_{I I}}\left(A \cap_{2} B\right) \subseteq_{2} \overline{N R S_{I I}}(A) \cap_{2} \overline{N R S_{I I}}(B)$.

Definition 5. [22] Suppose A, B are two NSs, then the Hamming distance between $A$ and $B$ is defined as:

$$
d_{N}(A, B)=\sum_{i=1}^{n}\left\{\left|T_{A}\left(x_{i}\right)-T_{B}\left(x_{i}\right)\right|+\left|I_{A}\left(x_{i}\right)-I_{B}\left(x_{i}\right)\right|+\left|F_{A}\left(x_{i}\right)-F_{B}\left(x_{i}\right)\right|\right\} .
$$

\section{Type-III NRS}

In this chapter, we introduce a new NRS, type-III NRS $\left(\mathrm{NRS}_{\text {III }}\right)$. We provide the differences between the three kinds of NRSs. The properties of $\mathrm{NRS}_{\text {III }}$ are also given.

Definition 6. Suppose (U, R) is an NAS. $\forall A \in N S(U)$, the type-III LUA of $A$, is defined as:

$$
\underline{N R S_{I I I}}(A)=\underset{y \in U}{\cap_{3}}\left(R^{c}(x, y) \cup_{3} A(y)\right), \overline{N R S_{I I I}}(A)=\underset{y \in U}{\cup_{3}}\left(R(x, y) \cap_{3} A(y)\right) .
$$

The pair $\left(\underline{N R S_{I I I}}(A), \overline{N R S_{I I I}}(A)\right)$ is called $\mathrm{NRS}_{\text {III }}$ of $A$.

Proposition 3. Suppose $(U, R)$ is an NAS. $\forall A, B \in N S(U)$, we have:

(1) If $A \subseteq_{3} B$, then $N R S_{I I I}(A) \subseteq_{3} N R S_{I I I}(B), \overline{N R S_{I I I}}(A) \subseteq_{3} \overline{N R S_{I I I}}(B)$.

(2) $N R S_{I I I}\left(A \cap_{3} B\right) \subseteq_{3} N R S_{I I I}(A) \cap_{3} N R S_{I I I}(B), N R S_{I I I}(A) \cup_{3} N R S_{I I I}(B) \subseteq_{3} N R S_{I I I}\left(A \cup_{3} B\right)$.

(3) $\overline{\overline{N R S_{I I I}}}\left(A \cap_{3} B\right) \subseteq_{3} \overline{\overline{N R S_{I I I}}}(A) \cap_{3} \overline{\overline{N R S_{I I I}}}(B), \overline{\overline{N R S_{I I I}}}(A) \cup_{3} \overline{\overline{N R S_{I I I}}}(B) \subseteq_{3} \overline{\overline{N R S_{I I I}}}\left(A \cup_{3} B\right)$.

Proof. (1) Assume $A \subseteq_{3} B$,

Case 1: If $T_{A}(x)<T_{B}(x), F_{A}(x) \geq F_{B}(x)$, then:

$$
\begin{aligned}
& T_{\underline{N R S_{I I I}}(A)}(x)=\underset{y \in U}{\wedge}\left[F_{R}(x, y) \vee T_{A}(y)\right] \leq \underset{y \in U}{\wedge}\left[F_{R}(x, y) \vee T_{B}(y)\right]=T_{\underline{N R S_{I I I}}(B)}(x) \\
& F_{\underline{N S_{I I I}}(A)}(x)=\underset{y \in U}{\vee}\left[T_{R}(x, y) \wedge F_{A}(y)\right] \geq \underset{y \in U}{\vee}\left[T_{R}(x, y) \wedge F_{B}(y)\right]=F_{\underline{N S_{I I I}}(B)}(x) .
\end{aligned}
$$


Hence,

$$
\underline{N R S_{I I I}}(A) \subseteq_{3} \underline{N R S_{I I I}}(B) .
$$

Case 2: If $T_{A}(x)=T_{B}(x), F_{A}(x)>F_{B}(x)$, then:

$$
\begin{aligned}
& T_{\underline{N R S_{I I I}}(A)}(x)=\underset{y \in U}{\wedge}\left[F_{R}(x, y) \vee T_{A}(y)\right]=\underset{y \in U}{\wedge}\left[F_{R}(x, y) \vee T_{B}(y)\right]=T_{\underline{N R S_{I I I}}(B)}(x) \\
& F_{\underline{N S_{I I I}}(A)}(x)=\underset{y \in U}{\vee}\left[T_{R}(x, y) \wedge F_{A}(y)\right] \geq \underset{y \in U}{\vee}\left[T_{R}(x, y) \wedge F_{B}(y)\right]=F_{\underline{N S_{I I I}}(B)}(x) .
\end{aligned}
$$

Hence,

$$
\operatorname{NRS}_{I I I}(A) \subseteq_{3} \underline{N R S_{I I I}}(B) .
$$

Case 3: suppose $T_{A}(x)=T_{B}(x), F_{A}(x)=F_{B}(x)$ and $I_{A}(x) \leq I_{B}(x)$, then:

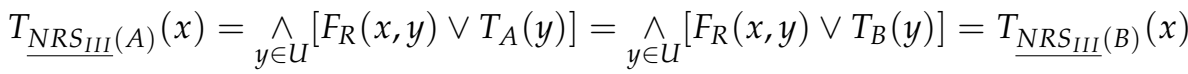

$$
\begin{aligned}
& F_{\text {NRS }_{I I I}(A)}(x)=\underset{y \in U}{\vee}\left[T_{R}(x, y) \wedge F_{A}(y)\right]=\underset{y \in U}{\vee}\left[T_{R}(x, y) \wedge F_{B}(y)\right]=F_{\underline{N S_{I I I}}(B)}(x) \\
& \underline{I_{N R S_{I I I}}(A)}(x)=\left\{\begin{array}{lc}
I_{A}\left(y_{j}\right), & R^{c}\left(x, y_{j}\right) \subseteq_{3} A\left(y_{j}\right) \subseteq_{3} A\left(y_{k}\right), y_{k}, y_{j} \in U \\
I_{R^{c}}\left(x, y_{j}\right), & A\left(y_{j}\right) \subseteq_{3} R^{c}\left(x, y_{j}\right) \\
1, & \text { else }
\end{array}\right. \\
& \underline{I_{\text {MNRS }_{I I I}}{ }^{(}(B)}(x)=\left\{\begin{array}{ll}
I_{B}\left(y_{j}\right), & R_{i}^{c}\left(x, y_{j}\right) \subseteq_{3} B\left(y_{j}\right) \subseteq_{3} B\left(y_{k}\right), y_{k}, y_{j} \in U \\
I_{R_{i}}\left(x, y_{j}\right), & B\left(y_{j}\right) \subseteq_{3} R_{i}^{c}\left(x, y_{j}\right) \\
1, & \text { else }
\end{array} .\right.
\end{aligned}
$$

Hence, $I_{N R S_{I I I}(A)}(x) \leq I_{N R S_{I I I}(B)}(x)$. So $\underline{N R S_{I I I}}(A) \subseteq_{3} \frac{N R S_{I I I}}{N R S_{I I I}}(B) \subseteq_{3}$.

Summing up the above, if $A \subseteq_{3} B$, then $\overline{N R S_{I I I}}(A) \subseteq_{3} \overline{N R S_{I I I}}(B)$.

Similarly, we can get $\overline{N R S_{I I I}}(A) \subseteq_{3} \overline{N R S_{I I I}(B)}$.

(2) According the Definition 6, we have:

$$
\begin{aligned}
& \underline{N R S_{I I I}}\left(A \cap_{3} B\right)=\underset{y \in U}{\cap_{3}}\left[R^{c}(x, y) \cup_{3}\left(A \cap_{3} B\right)(y)\right] \\
& \subseteq_{3}\left[\cap_{y \in U}\left(R^{c}(x, y) \cup_{3} A(y)\right)\right] \cap_{3}\left[\cap_{y \in U}\left(R^{c}(x, y) \cup_{3} B(y)\right)\right] \\
& =\underline{N R S_{I I I}}(A) \cap_{3} \underline{N R S_{I I I}}(B) .
\end{aligned}
$$

Similarly,

$$
\begin{aligned}
\underline{N R S_{I I I}}(A) \cup_{3} \underline{N R S_{I I I}}(B) & =\left[\underset{\cap_{3}}{\cap_{y \in U}}\left(R^{c}(x, y) \cup_{3} A(y)\right)\right] \cup_{3}\left[\underset{\cap_{3}}{\cap_{y \in U}}\left(R^{c}(x, y) \cup_{3} B(y)\right)\right] \\
& \subseteq_{3} \cap_{y \in U}\left[R^{c}(x, y) \cup_{3}\left(A \cup_{3} B\right)(y)\right] \\
& =\underline{N R S_{I I I}}\left(A \cup_{3} B\right) .
\end{aligned}
$$

(3) The proof is similar to that of Case 2.

Example 1. Define NAS $(U, R)$, where $U=\left\{x_{1}, x_{2}\right\}$ and $R$ is given in Table 1.

Table 1. A neutrosophic relation R.

\begin{tabular}{ccc}
\hline $\mathbf{R}$ & $\mathbf{x}_{\mathbf{1}}$ & $\mathbf{x}_{\mathbf{2}}$ \\
\hline $\mathrm{x}_{1}$ & $(0.4,0.6,0.7)$ & $(0.2,0.2,0.9)$ \\
$\mathrm{x}_{2}$ & $(0.7,0.1,0.4)$ & $(0.8,0.8,0.6)$ \\
\hline
\end{tabular}


Suppose $A$ is an NS and $A=\left\{\left(x_{1}, 0.8,0.2,0.1\right),\left(x_{2}, 0.4,0.9,0.5\right)\right.$. Then, by Definitions 3,4 and 6 , we can get:

$$
\begin{aligned}
& \underline{N R S_{I}}(A)\left(x_{1}\right)=(0.8,0.8,0.2), \underline{N R S_{I}}(A)\left(x_{2}\right)=(0.6,0.2,0.5), \\
& \overline{\overline{N R S_{I}}}(A)\left(x_{1}\right)=(0.4,0.6,0.7), \overline{\overline{N R S_{I}}}(A)\left(x_{2}\right)=(0.7,0.2,0.4) \text {, } \\
& \underline{N R S_{I I}}(A)\left(x_{1}\right)=(0.8,0.4,0.2), N_{N R S_{I I}}(A)\left(x_{2}\right)=(0.6,0.9,0.5) \text {, } \\
& \overline{\overline{N R S_{I I}}}(A)\left(x_{1}\right)=(0.4,0.2,0.7), \overline{\overline{N R S_{I I}}}(A)\left(x_{2}\right)=(0.7,0.8,0.4) \text {, } \\
& \operatorname{NRS}_{I I I}(A)\left(x_{1}\right)=(0.8,1,0.2), \underline{N R S_{I I I}}(A)\left(x_{2}\right)=(0.6,0,0.5), \\
& \overline{\operatorname{NRS}_{I I I}}(A)\left(x_{1}\right)=(0.4,0.6,0.7), \overline{\overline{N R S_{I I I}}}(A)\left(x_{2}\right)=(0.7,0.1,0.4) \text {. }
\end{aligned}
$$

\section{Type-I and Type-II MNRS}

We have proposed a kind of multigranulation neutrosophic rough set [30] (we called it type-I multigranulation neutrosophic rough set in this paper). $\mathrm{MNRS}_{\mathrm{I}}$ is based on a type-1 operator of NRs. In this chapter, we define the type-II multigranulation neutrosophic rough set $\left(\mathrm{MNRS}_{\mathrm{II}}\right)$, which is based on a type- 2 operator of NRs.

Definition 7. [28] Suppose $U$ is a non-empty finite universe, and $R_{i}(1 \leq i \leq m)$ is a binary NR on $U$. We call the tuple ordered set $\left(U, R_{i}\right)$ the multigranulation neutrosophic approximation space (MNAS).

Definition 8. [28] Suppose $\left(U, R_{i}\right)$ is an MNAS. $\forall A \in N S(U)$, the type-I optimistic LUA of $A$, represented by $\underline{\operatorname{MNRS}_{I}}{ }^{\circ}(A)$ and $\overline{\operatorname{MNRS}}_{I}{ }^{\circ}(A)$, is defined as:

$$
\begin{aligned}
& \underline{\operatorname{MNRS}}_{I}^{o}(A)(x)=\cup_{i=1}^{m}\left(\cap_{y \in U}\left(R_{i}^{c}(x, y) \cup_{1} A(y)\right)\right) \\
& {\overline{M N R S_{I}}}^{o}(A)(x)=\stackrel{\cap}{i=1}_{1}^{m}\left(\cup_{y \in U}\left(R_{i}(x, y) \cap_{1} A(y)\right)\right) .
\end{aligned}
$$

Then, $A$ is named a definable NS when $\operatorname{MNRS}_{I}{ }^{\circ}(A)={\overline{M N R S_{I}}}^{\circ}(A)$. Alternatively, we name the pair $\left(\underline{\operatorname{MNRS}}_{I}^{o}(A), \overline{\operatorname{MNRS}}_{I}^{o}(A)\right)$ an optimistic $\overline{\operatorname{MNRS}}_{\mathrm{I}}$.

Definition 9. [30] Suppose $\left(U, R_{i}\right)$ is an MNAS. $\forall A \in N S(U)$, the type-I pessimistic LUA of $A$, represented by $\operatorname{MNRS}_{I}^{p}(A)$ and $\overline{M N R S}_{I}^{p}(A)$, is defined as:

$$
\begin{aligned}
& \underline{\operatorname{MNRS}}^{p}(A)(x)=\cap_{i=1}^{m}\left(\bigcap_{y \in U}\left(R_{i}^{c}(x, y) \cup_{1} A(y)\right)\right) \\
& \overline{\operatorname{MNRS}}_{I}^{p}(A)(x)=\cup_{i=1}^{m}\left(\cup_{y \in U}\left(R_{i}(x, y) \cap_{1} A(y)\right)\right) .
\end{aligned}
$$

Similarly, $A$ is named a definable NS when ${\underline{M N R S_{I}}}^{p}(A)=\overline{\operatorname{MNRS}}_{I}^{p}(A)$. Alternatively, we name the pair $\left(\underline{\operatorname{MNRS}}_{I}^{p}(A), \overline{\operatorname{MNRS}}_{I}^{p}(A)\right)$ a pessimistic $\overline{\operatorname{MNRS}}_{\mathrm{I}}$.

Definition 10. Suppose $\left(U, R_{i}\right)$ is an MNAS. $\forall A \in N S(U)$, the type-II optimistic LUA of $A$, represented by ${\underline{M N R S_{I I}}}^{\circ}(A)$ and ${\overline{M N R S_{I I}}}^{\circ}(A)$, is defined as:

$$
\begin{aligned}
& \underline{\operatorname{MNRS}_{I I}}{ }^{o}(A)(x)=\cup_{i=1}^{m}\left(\cap_{y \in U}\left(R_{i}^{c}(x, y) \cup_{2} A(y)\right)\right) \\
& \overline{\operatorname{MNRS}}_{I I}{ }^{\circ}(A)(x)=\stackrel{\cap}{\cap}_{i=1}^{m}\left(\cup_{y \in U}\left(R_{i}(x, y) \cap_{2} A(y)\right)\right) .
\end{aligned}
$$


Then, $A$ is named a definable NS when $\operatorname{MNRS}_{I I}{ }^{\circ}(A)=\overline{\operatorname{MNRS}}_{I I}{ }^{\circ}(A)$. Alternatively, we name the pair $\left(\underline{\operatorname{MNRS}}_{I I}{ }^{\circ}(A), \overline{\operatorname{MNRS}}_{I I}{ }^{\circ}(A)\right)$ an optimistic $\mathrm{MNRS}_{\mathrm{II}}$.

Definition 11. Suppose $\left(U, R_{i}\right)$ is an MNAS. $\forall A \in N S(U)$, the type-II pessimistic LUA of $A$, represented by ${\underline{M N R S_{I I}}}^{p}(A)$ and ${\overline{M N R S_{I I}}}^{p}(A)$, is defined as:

$$
\begin{aligned}
& \underline{\operatorname{MNRS}}_{I I}^{p}(A)(x)=\stackrel{\cap}{\cap}_{i=1}^{m}\left(\bigcap_{y \in U}\left(R_{i}^{c}(x, y) \cup_{2} A(y)\right)\right) \\
& {\overline{M N R S_{I I}}}^{p}(A)(x)=\cup_{i=1}^{m}\left(\cup_{y \in U}\left(R_{i}(x, y) \cap_{2} A(y)\right)\right) .
\end{aligned}
$$

Similarly, $A$ is named a definable NS when $\operatorname{MNRS}_{I I}{ }^{p}(A)={\overline{M N R S_{I I}}}^{p}(A)$. Alternatively, we name the pair $\left(\underline{\operatorname{MNRS}}_{I I}^{p}(A), \overline{\operatorname{MNRS}}^{p}{ }^{p}(A)\right)$ a pessimistic MNRS $\mathrm{II}_{\text {. }}$.

Proposition 4. Suppose $\left(U, R_{i}\right)$ is an MNAS. $\forall A, B \in N S(U)$, then:

(1) $\operatorname{MNRS}_{I I}{ }^{o}(A)=\sim \overline{\operatorname{MNRS}}_{I I}^{o}(\sim A),{\overline{M N R S_{I I}}}^{p}(A)=\sim \overline{\operatorname{MNRS}}_{I I}^{p}(\sim A)$.

(2) ${\overline{\overline{\operatorname{MNRS}}_{I I}}}^{\circ}(A)=\sim{\operatorname{MNRS_{II}}}^{o}(\sim A),{\overline{\overline{M N R S_{I I}}}}^{p}(A)=\sim{\underline{M N R S_{I I}}}^{p}(\sim A)$.

(3) $\operatorname{MNRS}_{I I}^{o}\left(A \cap_{2} B\right)=\overline{M N R S}_{I I}^{o}(A) \cap_{2}{\underline{M N R S_{I I}}}^{o}(B), \underline{\operatorname{MNRS}}^{p}\left(A \cap_{2} B\right)=\operatorname{MNRS}_{I I}^{p}(A) \cap_{2}$ $\overline{\operatorname{MNRS}_{I I}}{ }^{p}(B)$.

(4) ${\overline{\overline{M N R S}_{I I}}}^{O}\left(A \cup_{2} B\right)=\overline{\operatorname{MNRS}}_{I I}^{o}(A) \cup_{2}{\overline{M N R S_{I I}}}^{o}(B),{\overline{M N R S_{I I}}}^{p}\left(A \cup_{2} B\right)=\overline{\operatorname{MNRS}}_{I I}^{p}(A) \cup_{2}$ $\overline{\operatorname{MNRS}}^{p}{ }^{p}(B)$.

(5) $\quad A \subseteq_{2} B \Rightarrow{\underline{M N R S_{I I}^{o}}}^{o}(A) \subseteq_{2} \underline{\operatorname{MNRS}}_{I I}^{o}(B), \underline{\operatorname{MNRS}}_{I I}^{p}(A) \subseteq_{2}{\underline{\operatorname{MNRS_{II}}}}^{p}(B)$.

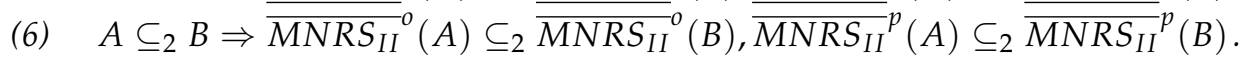

(7) ${\underline{M N R S_{I I}}}^{o}(A) \cup_{2} \operatorname{MNRS}_{I I}{ }^{o}(B) \subseteq_{2}{\underline{M N R S_{I I}}}^{o}\left(A \cup_{2} B\right), \operatorname{MNRS}_{I I}^{p}(A) \cup_{2}{\underline{M N R S_{I I}}}^{p}(B) \subseteq_{2}$ ${\overline{M N R S_{I I}}}^{p}\left(A \cup_{2} B\right)$.

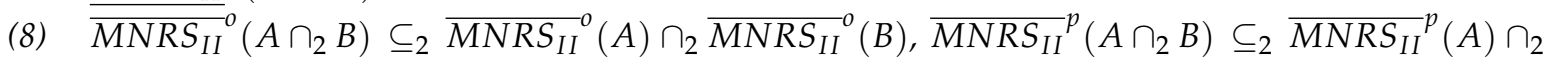
$\overline{\operatorname{MNRS}}_{I I}{ }^{p}(B)$.

Proof. Equations (1), (2), (5), and (6) are obviously according to Definitions 10 and 11. Next, we will prove Equations (3), (4), (7), and (8).

(3) By Definition 10,

$$
\begin{aligned}
& \underline{\operatorname{MNRS}}_{I I}^{o}\left(A \cap_{2} B\right)(x)=\cup_{i=1}^{m}\left(\bigcap_{y \in U}\left(R_{i}^{c}(x, y) \cup_{2}\left(A \cap_{2} B\right)(y)\right)\right) \\
& =\cup_{i=1}^{n}\left(\underset{y \in U}{\cap_{2}}\left(\left(R_{i}^{c}(x, y) \cup_{2} A(y)\right) \cap\left(R_{i}^{c}(x, y) \cup_{2} B(y)\right)\right)\right) \\
& =\left(\cup_{i=1}^{n}\left(\cap_{y \in U}\left(R_{i}^{c}(x, y) \cup_{2} A(y)\right)\right)\right) \cap_{2}\left(\cup_{i=1}^{n}\left(\cap_{y \in U}\left(R_{i}^{c}(x, y) \cup_{2} B(y)\right)\right)\right) \\
& =\underline{M N R S_{I I}} A(x) \cap_{2} \underline{M_{N R S_{I I}}} B(y) \text {. }
\end{aligned}
$$

Similarly, from Definition 11, we can get the following:

$$
\underline{\operatorname{MNRS}}_{I I}^{p}\left(A \cap_{2} B\right)=\underline{\operatorname{MNRS}_{I I}}{ }^{p}(A) \cap_{2} \underline{\operatorname{MNRS}}_{I I}^{p}(B) .
$$

(4) The proof is similar to that of Equation (3). 
(7) By Definition 10, we can get:

$$
\begin{aligned}
& T_{\underline{M N R S_{I I}{ }^{o}}\left(A \cup_{2} B\right)}(x)=m_{i=1}^{m} \operatorname{maxin}\left\{\max \left[F_{R_{i}}(x, y),\left(\max \left(T_{A}(y), T_{B}(y)\right)\right)\right]\right\} \\
& =\operatorname{maxmin}_{i=1}\left\{\max \left[\left(\max \left(F_{R_{i}}(x, y), T_{A}(y)\right)\right),\left(\max \left(F_{R_{i}}(x, y), T_{B}(y)\right)\right)\right]\right\} \\
& \geq \max \left\{\left[\operatorname{maxmin}_{i=1} \max _{y \in U}\left(\max \left(F_{R_{i}}(x, y), T_{A}(y)\right)\right)\right],\left[\operatorname{maxmin}_{i=1} \max \left(\max \left(F_{R_{i}}(x, y), T_{B}(y)\right)\right)\right]\right\} \\
& =\max \left({\underline{T N R S_{I I}{ }^{o}}}^{(A)}(x),{\underline{T N R S_{I I}{ }^{o}(B)}}(x)\right) \text {. }
\end{aligned}
$$

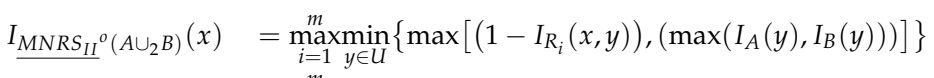

$$
\begin{aligned}
& =\operatorname{maxmin}_{i=1}^{m}\left\{\max \left[\left(\max \left(\left(1-I_{R_{i}}(x, y)\right), I_{A}(y)\right)\right),\left(\max \left(\left(1-I_{R_{i}}(x, y)\right), I_{B}(y)\right)\right)\right]\right\} \\
& \geq \max \left\{\left[\operatorname{maxmin}_{i=1}^{m}\left(\max \left(\left(1-I_{R_{i}}(x, y)\right), I_{A}(y)\right)\right)\right],\left[\operatorname{maxmin}_{i=1}^{m}\left(\max \left(\left(1-I_{R_{i}}(x, y)\right), I_{B}(y)\right)\right)\right]\right\} \\
& =\max \left({\underline{I_{M N R S_{I I}}^{o}(A)}}(x),{\underline{I_{M N R S_{I I}}^{o}(B)}}^{(x)}\right) \text {. } \\
& {\underline{F_{M N R S_{I I}}{ }^{o}\left(A \cup_{2} B\right)}}(x)=\operatorname{minmax}_{\substack{i=1 \\
m}}^{m}\left\{\min \left[T_{R_{i}}(x, y),\left(\min \left(F_{A}(y), F_{B}(y)\right)\right)\right]\right\} \\
& =\operatorname{minmax}_{i=1}^{m}\left\{\min \left[\min \left(T_{R_{i}}(x, y), F_{A}(y)\right)\right],\left[\min \left(T_{R_{i}}(x, y), F_{B}(y)\right)\right]\right\} \\
& \leq \min \left\{\left[\operatorname{minmax}_{i=1}^{m}\left(\min \left(T_{R_{i}}(x, y), F_{A}(y)\right)\right)\right],\left[\operatorname{minmax}_{i=1}^{m}\left(\min \left(T_{R_{i}}(x, y), F_{B}(y)\right)\right)\right]\right\} \\
& =\min \left(F_{\text {MNRS } S_{I I}{ }^{\circ}}(A)(x),{\underline{F_{M N R S_{I I}}{ }^{\circ}(B)}}(x)\right) \text {. }
\end{aligned}
$$

Hence, $\operatorname{MNRS}_{I I}{ }^{\circ}(A) \cup_{2} \operatorname{MNRS}_{I I}{ }^{\circ}(B) \subseteq_{2} \operatorname{MNRS}_{I I}{ }^{\circ}\left(A \cup_{2} B\right)$.

Additionally, according to Definition 11 , we can get ${\underline{M N R S_{I I}}}^{p}(A) \cup_{2}{\underline{M N R S_{I I}}}^{p}(B) \subseteq_{2}$ $M N S_{I I}{ }^{p}\left(A \cup \cup_{2} B\right)$.

(8) The proof is similar to that of Equation (7).

Remark 1. Note that if the NRs are the same one, then the optimistic (pessimistic) $\mathrm{MNRS}_{\text {II }}$ degenerates into $\mathrm{NRS}_{\text {II }}$ in Section 2.

\section{Type-III MNRS}

In this chapter, $\mathrm{MNRS}_{\text {III, }}$ which is based on a type-3 inclusion relation and corresponding union and intersection relations, is proposed and their characterizations are provided.

Definition 12. Suppose $\left(U, R_{i}\right)$ is an MNAS. $\forall A \in N S(U)$, the type-III optimistic LUA of $A$, represented by $\operatorname{MNRS}_{I I I}{ }^{\circ}(A)$ and $\overline{M N R S}_{I I I}{ }^{\circ}(A)$, is defined as:

$$
\begin{aligned}
& \frac{\operatorname{MNRS}_{I I I}{ }^{\circ}}{}(A)(x)=\cup_{i=1}^{m}\left(\bigcap_{y \in U}\left(R_{i}^{c}(x, y) \cup_{3} A(y)\right)\right) \\
& \overline{\operatorname{MNRS}}_{I I I}{ }^{\circ}(A)(x)=\stackrel{\cap}{i=1}_{3}^{m}\left(\cup_{y \in U}\left(R_{i}(x, y) \cap_{3} A(y)\right)\right) .
\end{aligned}
$$

Then, $A$ is named a definable NS when $\operatorname{MNRS}_{I I I}{ }^{\circ}(A)=\overline{\operatorname{MNRS}}_{I I I}{ }^{\circ}(A)$. Alternatively, we name the pair $\left(\underline{\operatorname{MNRS}}_{I I I}{ }^{\circ}(A), \overline{\operatorname{MNRS}}_{I I I}{ }^{\circ}(A)\right)$ an optimistic MNRS ${ }_{\text {IIII }}$.

Definition 13. Suppose $\left(U, R_{i}\right)$ is an MNAS. $\forall A \in N S(U)$, the type-III pessimistic LUA of $A$, represented by $\operatorname{MNRS}_{I I I}{ }^{p}(A)$ and $\overline{\operatorname{MNRS}}_{I I I}{ }^{p}(A)$, is defined as:

$$
\underline{\operatorname{MNRS}_{I I I}}{ }^{p}(A)(x)=\stackrel{\cap}{n}_{i=1}^{m}\left(\cap_{y \in U}\left(R_{i}^{c}(x, y) \cup_{3} A(y)\right)\right)
$$




$$
\overline{\operatorname{MNRS}}_{I I I} p(A)(x)=\cup_{i=1}^{m}\left(\cup_{y \in U}\left(R_{i}(x, y) \cap_{3} A(y)\right)\right) .
$$

Similarly, $A$ is named a definable NS when $\underline{\operatorname{MNRS}}_{I I I}{ }^{p}(A)=\overline{\operatorname{MNRS}}_{I I I}{ }^{p}(A)$. Alternatively, we name the pair $\left(\underline{\operatorname{MNRS}}_{I I I}{ }^{p}(A), \overline{\operatorname{MNRS}}_{I I I}^{p}(A)\right)$ a pessimistic MNRS ${ }_{\text {IIII }}$.

Proposition 5. Suppose $\left(U, R_{i}\right)$ is an MNAS. $\forall A, B \in N S(U)$, then:

(1) $\operatorname{MNRS}_{I I I}{ }^{o}(A)=\sim \overline{\operatorname{MNRS}}_{I I I}^{o}(\sim A), \operatorname{MNRS}_{I I I}^{p}(A)=\sim \overline{\operatorname{MNRS}}_{I I I}^{p}(\sim A)$.

(2) ${\overline{\overline{M N R S}_{I I I}}}^{o}(A)=\sim \operatorname{MNRS}_{I I I}{ }^{\circ}(\sim A),{\overline{\overline{M N R S_{I I I}}}}^{p}(A)=\sim \operatorname{MNRS}_{I I I}^{p}(\sim A)$.

(3) $\quad A \subseteq_{3} B \Rightarrow \operatorname{MNRS}_{I I I}{ }^{o}(A) \subseteq_{3} \operatorname{MNRS}_{I I I}{ }^{o}(B), \operatorname{MNRS}_{I I I}{ }^{p}(A) \subseteq_{3} \operatorname{MNRS}_{I I I}{ }^{p}(B)$.

(4) $\quad A \subseteq_{3} B \Rightarrow{\overline{\overline{M N R S}_{I I I}}}^{o}(A) \subseteq_{3}{\overline{\overline{M N R S_{I I I}}}}^{o}(B),{\overline{\overline{M N R S_{I I}}}}^{p}(A) \subseteq_{3}{\overline{\overline{M N R S_{I I I}}}}^{p}(B)$.

(5) $\operatorname{MNRS}_{I I I}{ }^{\circ}\left(A \cap_{3} B\right) \quad \subseteq_{3} \quad \operatorname{MNRS}_{I I I}{ }^{\circ}(A) \cap_{3}{\underline{M N R S_{I I I}}}^{\circ}(B), \quad \operatorname{MNRS}_{I I I}^{p}\left(A \cap_{3} B\right) \quad \subseteq_{3}$ $\overline{\operatorname{MNRS}}_{I I I} p(A) \cap_{3} \operatorname{MNRS}_{I I I} p(B)$.

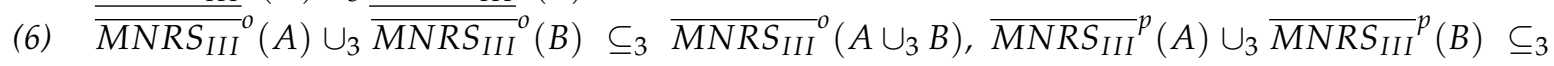
$\overline{\operatorname{MNRS}}_{I I I}^{p}\left(A \cup_{3} B\right)$.

(7) $\operatorname{MNRS}_{I I I}{ }^{o}(A) \cup_{3}{\underline{M N R S_{I I I}}}^{o}(B) \subseteq_{3} \underline{\operatorname{MNRS}}_{I I I}{ }^{o}\left(A \cup_{3} B\right), \underline{M N R S}_{I I I}^{p}(A) \cup_{3} \underline{\operatorname{MNRS}}_{I I I}^{p}(B) \subseteq_{3}$ $\overline{M N R S}_{I I I} p(A \cup 3)$.

(8) ${\overline{\overline{M N R S_{I I I}}}}^{O}\left(A \cap_{3} B\right) \quad \subseteq_{3} \quad{\overline{\operatorname{MNRS_{III}}}}^{O}(A) \cap_{3}{\overline{\operatorname{MNRS_{III}}}}^{o}(B), \quad{\overline{\operatorname{MNRS_{III}}}}^{p}\left(A \cap_{3} B\right) \quad \subseteq_{3}$ $\overline{\operatorname{MNRS}}_{I I I}{ }^{p}(A) \cap_{3} \overline{\operatorname{MNRS}}_{I I I}^{p}(B)$.

Proof. Equations (1) and (2) can be directly derived from Definitions 12 and 13 . We only provide the proof of Equations (3)-(8).

(3) Suppose $A \subseteq_{3} B$, then:

Case 1: If $T_{A}(x)<T_{B}(x), F_{A}(x) \geq F_{B}(x)$, then:

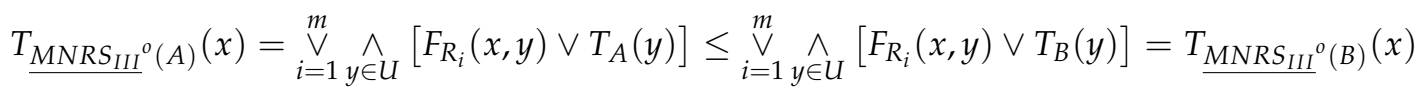

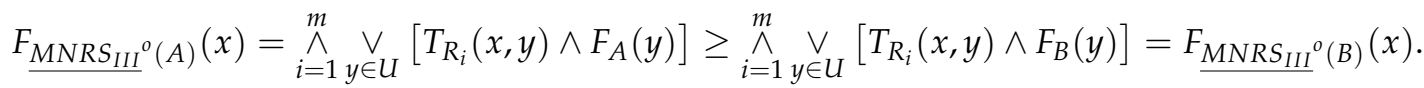

Hence, $\operatorname{MNRS}_{I I I}{ }^{\circ}(A) \subseteq_{3} \operatorname{MNRS}_{I I I}{ }^{\circ}(B)$.

Case 2: If $T_{A}(x)=T_{B}(x), F_{A}(x)>F_{B}(x)$, then:

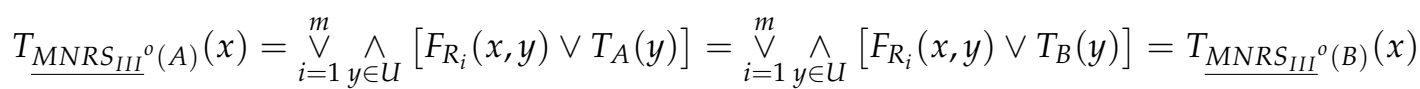

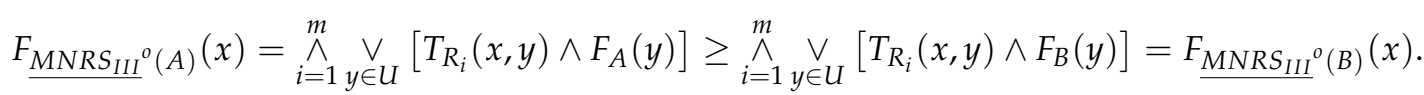

Hence, $\operatorname{MNRS}_{I I I}{ }^{\circ}(A) \subseteq_{3} \operatorname{MNRS}_{I I I}{ }^{\circ}(B)$.

Case 3: suppose $T_{A}(x)=\widehat{T_{B}(x), F_{A}(x)}=F_{B}(x)$ and $I_{A}(x) \leq I_{B}(x)$, then:

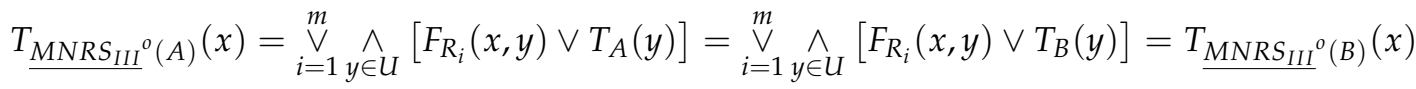

$$
\begin{aligned}
& F_{\text {MNRS }_{I I I}{ }^{o}(A)}(x)=\wedge_{i=1}^{m} \vee_{y \in U}^{\vee}\left[T_{R_{i}}(x, y) \wedge F_{A}(y)\right] \geq \wedge_{i=1}^{m} \underset{y \in U}{\vee}\left[T_{R_{i}}(x, y) \wedge F_{B}(y)\right]=F_{\text {MNRS }_{I I I}^{o}(B)}(x)
\end{aligned}
$$

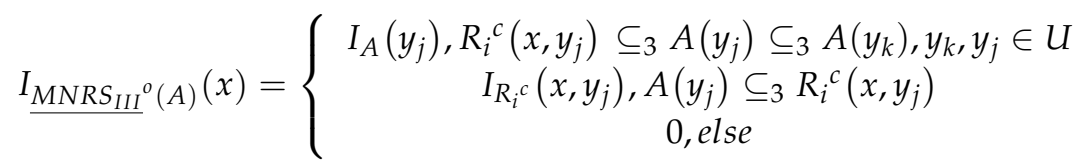




$$
\underline{I_{\operatorname{MNRS}_{I I I}}{ }^{(}(B)}(x)=\left\{\begin{array}{c}
I_{B}\left(y_{j}\right), R_{i}^{c}\left(x, y_{j}\right) \subseteq_{3} B\left(y_{j}\right) \subseteq_{3} B\left(y_{k}\right), y_{k}, y_{j} \in U \\
I_{R_{i}}{ }^{c}\left(x, y_{j}\right), B\left(y_{j}\right) \subseteq_{3} R_{i}^{c}\left(x, y_{j}\right) \\
0, \text { else }
\end{array}\right.
$$

Hence, $I_{M N R S_{I I I}{ }^{o}(A)}(x) \leq I_{M N R S_{I I I}{ }^{o}(B)}(x)$. So, $\operatorname{MNRS}_{I I I}{ }^{o}(A) \subseteq_{3} \operatorname{MNRS}_{I I I}{ }^{o}(B)$.

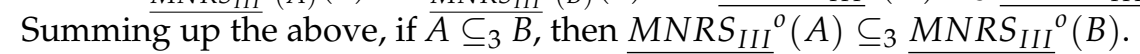

Similarly, we can get $\operatorname{MNRS}_{I I I}{ }^{p}(A) \subseteq_{3}{\overline{M N R S_{I I I}}}^{p}(B)$.

(4) The proof is similar to that of Equation (3).

(5) From Definition 12, we have:

$$
\begin{aligned}
& \underline{\operatorname{MNRS}}_{I I I}{ }^{o}\left(A \cap_{3} B\right)=\cup_{i=1}^{m}\left(\cap_{y \in U}\left(R_{i}{ }^{c}(x, y) \cup_{3}\left(A(y) \cap_{3} B(y)\right)\right)\right) \\
& \subseteq_{3} \cup_{i=1}^{m}\left(\cap_{y \in U}\left(\left(R_{i}^{c}(x, y) \cup_{3} A(y)\right) \cap_{3}\left(R_{i}^{c}(x, y) \cup_{3} B(y)\right)\right)\right) \\
& \subseteq_{3}\left(\cup_{i=1}^{m}\left(\cap_{y \in U}\left(\cap_{i}^{c}(x, y) \cup_{3} A(y)\right)\right)\right) \cap_{3}\left(\cup_{i=1}^{m}\left(\cap_{y \in U}\left(R_{i}^{c}(x, y) \cup_{3} B(y)\right)\right)\right) \\
& =\underline{\operatorname{MNRS}_{I I I}}{ }^{\circ}(A) \cap_{3} \underline{\operatorname{MNRS}_{I I I}}{ }^{\circ}(B) .
\end{aligned}
$$

Similarly, from Definition 13, we can get $\underline{\operatorname{MNRS}}_{I I I}^{p}\left(A \cap_{3} B\right) \subseteq_{3} \underline{\operatorname{MNRS}}_{I I I}^{p}(A) \cap_{3} \underline{\operatorname{MNRS}}_{I I I}{ }^{p}(B)$.

(6) From Definition 12, we have:

$$
\begin{aligned}
& \overline{\operatorname{MNRS}}_{I I I}{ }^{o}(A) \cup_{3}{\overline{\operatorname{MNRS_{III}}}}^{o}(B)=\left(\bigcap_{i=1}^{m}\left(\bigcup_{y \in U}\left(R_{i}(x, y) \cap_{3} A(y)\right)\right)\right) \cup_{3}\left(\bigcap_{i=1}^{m}\left(\cup_{y \in U}\left(R_{i}(x, y) \cap_{3} B(y)\right)\right)\right) \\
& \subseteq_{3} \cap_{i=1}^{m}\left(\cup_{y \in U}\left(\left(R_{i}(x, y) \cap_{3} A(y)\right) \cup_{3}\left(R_{i}(x, y) \cap_{3} B(y)\right)\right)\right) \\
& \subseteq_{3} \stackrel{m}{n}_{i=1}^{m}\left(\cup_{y \in U}\left(R_{i}(x, y) \cap_{3}\left(A(y) \cup_{3} B(y)\right)\right)\right) \\
& ={\overline{M N R S_{I I I}}}^{\circ}\left(A \cup_{3} B\right) \text {. }
\end{aligned}
$$

Similarly, from Definition 13, we can get $\overline{\operatorname{MNRS}}_{I I I}^{p}\left(A \cup_{3} B\right)=\overline{\operatorname{MNRS}}_{I I I}^{p}(A) \cup_{3} \overline{\operatorname{MNRS}}_{I I I}^{p}(B)$.

(7) From Definition 12, we have:

$$
\begin{aligned}
& \underline{\operatorname{MNRS}_{I I I}{ }^{o}}\left(A \cup_{3} B\right)=\cup_{i=1}^{m}\left(\cap_{y \in U}\left(R_{i}^{c}(x, y) \cup_{3}\left(A \cup_{3} B\right)(y)\right)\right) \\
& =\cup_{i=1}^{m}\left(\underset{y \in U}{\cap_{3}}\left(R_{i}^{c}(x, y) \cup_{3}\left(A(y) \cup_{3} B(y)\right)\right)\right) \\
& \supseteq_{3} \cup_{i=1}^{m}\left(\left(\left[\cap_{y \in U}\left(R_{i}^{c}(x, y) \cup_{3} A(y)\right)\right] \cup_{3}\left[\bigcap_{y \in U}\left(R_{i}^{c}(x, y) \cup_{3} B(y)\right)\right]\right)\right) \\
& =\left(\cup_{i=1}^{m}\left[\cap_{y \in U}\left(R_{i}^{c}(x, y) \cup_{3} A(y)\right)\right]\right) \cup_{3}\left(\cup_{i=1}^{m}\left[\cap_{y \in U}\left(R_{i}^{c}(x, y) \cup_{3} B(y)\right)\right]\right) \\
& =\underline{\operatorname{MNRS}_{I I I}}{ }^{\circ}(A) \cup_{3} \underline{\operatorname{MNRS}_{I I I}}{ }^{\circ}(B) \text {. }
\end{aligned}
$$

Hence, $\operatorname{MNRS}_{I I I}{ }^{\circ}(A) \cup_{3} \underline{M N R S}_{I I I}{ }^{\circ}(B) \subseteq_{3} \underline{M N R S_{I I I}}\left(A \cup_{3} B\right)$.

Additionally, from Definition 13 , we can get $\operatorname{MNRS}_{I I I}^{p}(A) \cup_{3} \operatorname{MNRS}_{I I I}^{p}(B) \subseteq_{3}$ $\operatorname{MNRS}_{I I I}{ }^{p}\left(A \cup_{3} B\right)$. 
(8) From Definition 12, we have:

$\overline{\operatorname{MNRS}}_{I I I}{ }^{\circ}\left(A \cap \cap_{3} B\right)$

$$
\begin{aligned}
& =\stackrel{m}{\cap}_{i=1}^{m}\left(\cup_{y \in U}\left(R_{i}(x, y) \cap_{3}\left(A \cap_{3} B\right)(y)\right)\right) \\
& =\cap_{i=1}^{m}\left(\cup_{y \in U}\left(R_{i}(x, y) \cap_{3}\left(A(y) \cap_{3} B(y)\right)\right)\right) \\
& \subseteq_{3} \cap_{i=1}^{m}\left(\left[\underset{y \in U}{\cup_{2}}\left(R_{i}(x, y) \cap_{3} A(y)\right)\right] \cap_{3}\left[\underset{y \in U}{\cup_{3}}\left(R_{i}(x, y) \cap_{3} B(y)\right)\right]\right)
\end{aligned}
$$

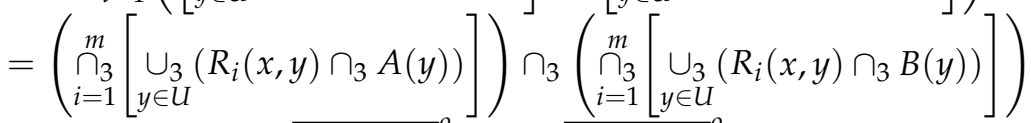

$$
\begin{aligned}
& ={\overline{M N R S_{I I I}}}^{\circ}(A) \cap_{3}{\overline{\operatorname{MNRS}_{I I I}}}^{\circ}(B) .
\end{aligned}
$$

Hence, $\overline{\operatorname{MNRS}}_{I I I}{ }^{\circ}\left(A \cap_{3} B\right) \subseteq_{3} \overline{\operatorname{MNRS}}_{I I I}{ }^{\circ}(A) \cap_{3} \overline{\operatorname{MNRS}}_{I I I}{ }^{\circ}(B)$.

Similarly, from Definition 13, we can get $\overline{\operatorname{MNRS}}_{\text {III }}^{p}\left(A \cap_{3} B\right) \subseteq_{3}{\overline{\operatorname{MNRS_{III}}}}^{p}(A) \cap_{3} \overline{\operatorname{MNRS}}_{I I I}^{p}(B)$.

Remark 2. Note that if the NRs are the same one, then the optimistic (pessimistic) $\mathrm{MNRS}_{\text {III }}$ degenerates into $\mathrm{NRS}_{\text {III }}$ in Section 3.

\section{Type-III MNRS in Two Universes with Its Applications}

In this chapter, we propose the concept of $\mathrm{MNRS}_{\text {III }}$ in two universes and use it to deal with the MAGDM problem.

Definition 14. [28] Suppose $U, V$ are two non-empty finite universes, and $R_{i} \in N S(U \times V)(1 \leq i \leq m)$ is a binary NR. We call $\left(U, V, R_{i}\right)$ the MNAS in two universes.

Definition 15. Suppose $\left(U, V, R_{i}\right)$ is an MNAS in two universes. $\forall A \in N S(V)$ and $x \in U$, the type-III optimistic LUA of $A$ in $\left(U, V, R_{i}\right)$, represented by ${\operatorname{MNRS_{III}}}^{\circ}(A)$ and ${\overline{M N R S_{I I I}}}^{\circ}(A)$, is defined as:

$$
\begin{aligned}
& {\operatorname{MNRS_{III}}}^{o}(A)(x)=\cup_{i=1}^{m}\left(\bigcap_{y \in V}\left(R_{i}^{c}(x, y) \cup_{3} A(y)\right)\right) \\
& \overline{\operatorname{MNRS}}_{I I I}{ }^{\circ}(A)(x)=\overbrace{i=1}^{m}\left(\cup_{y \in V}\left(R_{i}(x, y) \cap_{3} A(y)\right)\right) .
\end{aligned}
$$

Then, $A$ is named a definable NS in two universes when $\operatorname{MNRS}_{I I I}{ }^{o}(A)=\overline{\operatorname{MNRS}}_{I I I}{ }^{o}(A)$. Alternatively, we name the pair $\left(\underline{\operatorname{MNRS}}_{I I I}{ }^{\circ}(A), \overline{\operatorname{MNRS}}_{I I I}{ }^{\circ}(A)\right)$ an optimistic MNRS $S_{\text {III }}$ in two universes.

Definition 16. Suppose $\left(U, V, R_{i}\right)$ is an MNAS in two universes. $\forall A \in N S(V)$ and $x \in U$, the type-III pessimistic LUA of $A$ in $\left(U, V, R_{i}\right)$, denoted by ${\underline{M N R S_{I I I}}}^{p}(A)$ and $\overline{\operatorname{MNRS}}_{I I I}^{p}(A)$, is defined as follows:

$$
\begin{aligned}
& \operatorname{MNRS}_{I I I}^{p}(A)(x)=\overbrace{i=1}^{m}\left(\cap_{y \in V}\left(R_{i}^{c}(x, y) \cup_{3} A(y)\right)\right) \\
& \overline{\operatorname{MNRS}}_{I I I}^{p}(A)(x)=\cup_{i=1}^{m}\left(\cup_{y \in V}\left(R_{i}(x, y) \cap_{3} A(y)\right)\right) .
\end{aligned}
$$

Similarly, $A$ is named a definable NS when $\operatorname{MNRS}_{I I I}{ }^{p}(A)=\overline{\operatorname{MNRS}}_{I I I}^{p}(A)$. Alternatively, we name the pair $\left(\underline{\operatorname{MNRS}}_{I I I}{ }^{p}(A), \overline{\operatorname{MNRS}}_{I I I}^{p}(A)\right)$ a pessimistic MNRS ${ }_{\text {III }}$ in two universes. 
Remark 3. Note that if the two domains are the same, then the optimistic (pessimistic) $\mathrm{MNRS}_{\text {III }}$ in two universes degenerates into the optimistic (pessimistic) $\mathrm{MNRS}_{\text {III }}$ in a single universe in Section 5.

The MAGDM problem is becoming more and more generally present in our daily life. MAGDM means to select or rank all the feasible alternatives in various criterions. There are many ways to solve the MAGDM problem, but we use MNRS to solve it in this paper. Next, we give the basic description of the considered MAGDM problem.

For the car-ranking question, suppose $U=\left\{x_{1}, x_{2}, \ldots, x_{n}\right\}$ is the decision set and $V=\left\{y_{1}, y_{2}, \ldots\right.$, $\left.y_{m}\right\}$ is the criteria set in which $x_{1}$ represents "very popular", $x_{2}$ represents "popular", $x_{3}$ represents "less popular" $, \ldots, x_{n}$ represents "not popular", $y_{1}$ represents the vehicle type", $y_{2}$ represents the size of the space, $y_{3}$ represents the ride height, $y_{4}$ represents quality, and $\ldots, y_{m}$ represents length of durability. Then, $l$ selection experts make evaluations about the criteria sets according to their own experiences. Here, the evaluations were shown by NRs. Next, we calculate the degree of popularity for a given car. Therefore, we need to use MGNRS to solve the above problem. For the MAGDM problem under a multigranulation neutrosophic environment, the optimistic lower approximation can be regarded as an optimistic risk decision, and the optimistic upper approximation can be regarded as an optimistic conservative decision. Additionally, the pessimistic lower approximation can be regarded as a pessimistic risk decision and the pessimistic upper approximation can be regarded as a pessimistic conservative decision. According to the distance of neutrosophic sets, we define the difference function $d_{N}(A, B)\left(x_{i}\right)=(1 / 3)\left(\left|T_{A}\left(x_{i}\right)-T_{B}\left(x_{i}\right)\right|+\left|I_{A}\left(x_{i}\right)-I_{B}\left(x_{i}\right)\right|+\left|F_{A}\left(x_{i}\right)-F_{B}\left(x_{i}\right)\right|\right)$. We used the difference function to represent the distance of optimistic (pessimistic) upper and lower approximation. The smaller the value of the distance is, the better the alternative $x_{i}$ is, because the risk decision and the conservative decision are close. By comparing the distance value, all alternatives can be ranked and we can choose the optimal alternative. In this paper, we only used three kinds of optimistic upper and lower approximation to decision-making.

Next, we show the process of the above car-ranking question based on MGNRSs over two universes. Let $R_{l} \in N R(U \times V)$ be NRs from $U$ to $V$, where $\forall\left(x_{i}, y_{j}\right) \in U \times V, R_{l}\left(x_{i}, y_{j}\right)$ denotes the degree of popularity for criteria set $y_{j}\left(y_{j} \in V\right)$. $R_{l}$ can be obtained according to experts' experience. Given a car $A$, according to the unconventional questionnaire (suppose there are three options-"like", "not like", and "neutral" to choose for each of the criteria sets, and everyone can choose one or more options), then we can get the popularity of every criterion as described by an NS $A$ in the universe $V$ according to the questionnaire. By use of the following Algorithm 1, we can determine the degree of popularity of the given car $A$.

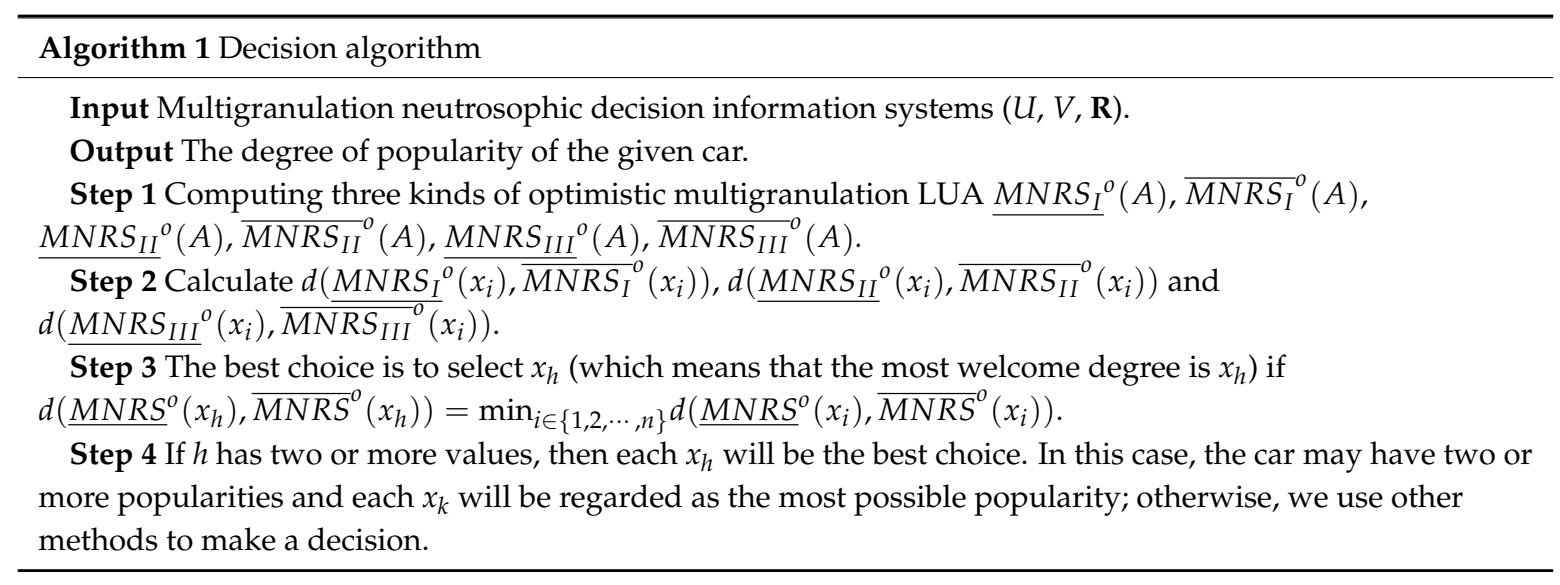

Next, we use an example to explain the algorithm.

Let $U=\left\{x_{1}, x_{2}, x_{3}, x_{4}\right\}$ be the decision set, in which $x_{1}$ denotes "very popular", $x_{2}$ denotes "popular", $x_{3}$ denotes "less popular", and $x_{4}$ denotes "not popular". Let $V=\left\{y_{1}, y_{2}, y_{3}, y_{4}, y_{5}\right\}$ be 
criteria sets, in which $y_{1}$ denotes the vehicle type, $y_{2}$ denotes the size of the space, $y_{3}$ denotes the ride height, $y_{4}$ denotes quality, and $y_{5}$ denotes length of durability.

Suppose that $R_{1}, R_{2}$, and $R_{3}$ are given by three invited experts. They provide their evaluations for all criteria $y_{j}$ with respect to decision set elements $x_{i}$. The evaluation $R_{1}, R_{2}$, and $R_{3}$ are NRs between attribute set $V$ and decision evaluation set $U$., that is., there are $R_{1}, R_{2}, R_{3} \in N R(U \times V)$.

Suppose three experts present their judgment (the neutrosophic relation $R_{1}, R_{2}$, and $R_{3}$ ) for the attribute and decision sets in Tables 2-4:

Table 2. Neutrosophic relation $R_{1}$.

\begin{tabular}{llllll}
\hline $\boldsymbol{R}_{\mathbf{1}}$ & $y_{\mathbf{1}}$ & $\mathbf{y}_{\mathbf{2}}$ & $\boldsymbol{y}_{\mathbf{3}}$ & $\boldsymbol{y}_{\mathbf{4}}$ & $\mathbf{y}_{\mathbf{5}}$ \\
\hline$x_{1}$ & $(0.8,0.6,0.5)$ & $(0.2,0.3,0.9)$ & $(0,0,1)$ & $(0.7,0.5,0.6)$ & $(0,0,1)$ \\
$x_{2}$ & $(0.6,0.4,0.6)$ & $(0.9,0.3,0.4)$ & $(1,0,0)$ & $(0,0,1)$ & $(0.3,0.6,0.7)$ \\
$x_{3}$ & $(0.2,0.5,0.9)$ & $(0.6,0.7,0.5)$ & $(0.8,0.7,0.8)$ & $(0,0,1)$ & $(1,0,0)$ \\
$x_{4}$ & $(0.6,0.4,0.7)$ & $(0,0,1)$ & $(0,0,1)$ & $(0.9,0.8,0.1)$ & $(0,0,1)$ \\
\hline
\end{tabular}

Table 3. Neutrosophic relation $R_{2}$.

\begin{tabular}{llllll}
\hline $\boldsymbol{R}_{\mathbf{2}}$ & $\boldsymbol{y}_{\mathbf{1}}$ & $\boldsymbol{y}_{\mathbf{2}}$ & $\boldsymbol{y}_{\mathbf{3}}$ & $\boldsymbol{y}_{\mathbf{4}}$ & $\boldsymbol{y}_{\mathbf{5}}$ \\
\hline$x_{1}$ & $(0.9,0.3,0.6)$ & $(0,0,1)$ & $(0,0,1)$ & $(0.5,0.6,0.5)$ & $(0.2,0.3,0.9)$ \\
$x_{2}$ & $(0.3,0.7,0.8)$ & $(0.7,0.5,0.6)$ & $(0.9,0.1,0.1)$ & $(0,0,1)$ & $(0.4,0.5,0.8)$ \\
$x_{3}$ & $(0.1,0.6,0.8)$ & $(0.3,0.6,0.5)$ & $(0.7,0.3,0.6)$ & $(0,0,1)$ & $(1,0,0)$ \\
$x_{4}$ & $(0.7,0.5,0.6)$ & $(0,0,1)$ & $(0,0,1)$ & $(1,0,0)$ & $(0,0,1)$ \\
\hline
\end{tabular}

Table 4. Neutrosophic relation $R_{3}$.

\begin{tabular}{llllll}
\hline $\boldsymbol{R}_{\mathbf{3}}$ & $\boldsymbol{y}_{\mathbf{1}}$ & $\boldsymbol{y}_{\mathbf{2}}$ & $\boldsymbol{y}_{\mathbf{3}}$ & $\boldsymbol{y}_{\mathbf{4}}$ & $\boldsymbol{y}_{\mathbf{5}}$ \\
\hline$x_{1}$ & $(0.6,0.9,0.4)$ & $(0.1,0.1,0.8)$ & $(0.1,0,0.9)$ & $(0.8,0.4,0.8)$ & $(0,0,1)$ \\
$x_{2}$ & $(0.5,0.6,0.6)$ & $(0.6,0.2,0.7)$ & $(1,0,0)$ & $(0,0,1)$ & $(0,0,1)$ \\
$x_{3}$ & $(0.1,0.4,0.7)$ & $(0.2,0.2,0.7)$ & $(0.5,0.7,0.6)$ & $(0,0,1)$ & $(0.9,0.1,0.2)$ \\
$x_{4}$ & $(0.6,0.3,0.4)$ & $(0,0,1)$ & $(0,0,1)$ & $(0.7,0.5,0.4)$ & $(0,0,1)$ \\
\hline
\end{tabular}

Suppose $A$ is a car and each criterion in $V$ is as follows:

$$
A=\left\{\left(y_{1}, 0.9,0.2,0.2\right),\left(y_{2}, 0.2,0.7,0.8\right),\left(y_{3}, 0,1,0.3\right),\left(y_{4}, 0.7,0.6,0.3\right),\left(y_{5}, 0.1,0.8,0.9\right)\right\} .
$$

Then, we can calculate the three kinds of optimistic LUAs of $A$ as follow:

$$
\begin{aligned}
& \operatorname{MNRS}_{I}{ }^{o}(A)\left(x_{1}\right)=(0.8,1,0.3), \operatorname{MNRS}_{I}^{o}(A)\left(x_{2}\right)=(0.1,0.9,0.6), \\
& {\overline{M N R S_{I}}}^{\circ}(A)\left(x_{3}\right)=(0.2,0.8,0.9), \operatorname{MNRS}_{I}^{o}(A)\left(x_{4}\right)=(0.7,1,0.3) \text {, } \\
& \overline{\operatorname{MNRS}}_{I}^{\circ}(A)\left(x_{1}\right)=(0.7,0.6,0.5),{\overline{\operatorname{MNRS_{I}}}}^{\circ}(A)\left(x_{2}\right)=(0.3,0.6,0.3) \text {, } \\
& \overline{\operatorname{MNRS}}_{I}{ }^{\circ}(A)\left(x_{3}\right)=(0.2,0.6,0.8), \overline{\operatorname{MNRS}}_{I}{ }^{\circ}(A)\left(x_{4}\right)=(0.7,0.5,0.4) \text {, } \\
& \operatorname{MNRS}_{I I}{ }^{o}(A)\left(x_{1}\right)=(0.8,0.6,0.3), \operatorname{MNRS}_{I I}{ }^{\circ}(A)\left(x_{2}\right)=(0.1,0.6,0.6), \\
& {\overline{\operatorname{MNRS}_{I I}}}^{\circ}(A)\left(x_{3}\right)=(0.2,0.6,0.9),{\overline{M N R S_{I I}}}^{\circ}(A)\left(x_{4}\right)=(0.7,0.6,0.3) \text {, } \\
& {\overline{\overline{M N R S_{I I}}}}^{\circ}(A)\left(x_{1}\right)=(0.7,0.4,0.5),{\overline{\overline{M N R S}_{I I}}}^{\circ}(A)\left(x_{2}\right)=(0.3,0.2,0.3) \text {, } \\
& \overline{\operatorname{MNRS}}_{I I}{ }^{\circ}(A)\left(x_{3}\right)=(0.2,0.6,0.8), \overline{\operatorname{MNRS}}_{I I}{ }^{\circ}(A)\left(x_{4}\right)=(0.7,0.2,0.4) \text {, } \\
& \operatorname{MNRS}_{I I I}{ }^{\circ}(A)\left(x_{1}\right)=(0.8,0,0.3), \operatorname{MNRS}_{I I I}{ }^{\circ}(A)\left(x_{2}\right)=(0.1,0,0.6) \text {, } \\
& {\overline{M N R S_{I I I}}}^{\circ}(A)\left(x_{3}\right)=(0.2,0.9,0.9),{\overline{M N R S_{I I I}}}^{\circ}(A)\left(x_{4}\right)=(0.7,0.6,0.3) \text {, } \\
& {\overline{\operatorname{MNRS}_{I I I}}}^{\circ}(A)\left(x_{1}\right)=(0.7,1,0.5),{\overline{\overline{M N R S_{I I I}}}}^{\circ}(A)\left(x_{2}\right)=(0.3,0,0.3) \text {, } \\
& \overline{\operatorname{MNRS}}_{I I I}{ }^{\circ}(A)\left(x_{3}\right)=(0.2,0.7,0.8){\overline{M N R S_{I I I}}}^{\circ}(A)\left(x_{4}\right)=(0.7,0.5,0.4) \text {. }
\end{aligned}
$$


12. Wang, H.B.; Smarandache, F.; Zhang, Y.; Sunderraman, R. Single valued neutrosophic sets. Multispace Multistructure 2010, 4, 410-413.

13. Singh, P.K. Three-way fuzzy concept lattice representation using neutrosophic set. Int. J. Mach. Learn. Cybern. 2017, 8, 69-79. [CrossRef]

14. Peng, X.; Liu, C. Algorithms for neutrosophic soft decision making based on EDAS, new similarity measure and level soft set. J. Intell. Fuzzy Syst. 2017, 32, 955-968. [CrossRef]

15. Ye, J. A multicriteria decision-making method using aggregation operators for simplified neutrosophic sets. J. Intell. Fuzzy Syst. 2014, 26, 2459-2466.

16. Zhang, X.; Bo, C.X.; Smarandache, F.; Dai, J.H. New inclusion relation of neutrosophic sets with applications and related lattice structure. Int. J. Mach. Learn. Cybern. 2018, 9, 1753-1763. [CrossRef]

17. Dubois, D.; Prade, H. Rough fuzzy sets and fuzzy rough sets. Inter. J. General Syst. 1990, 17, $191-209$. [CrossRef]

18. Broumi, S.; Smarandache, F.; Dhar, M. Rough neutrosophic sets. Neut. Sets Syst. 2014, 3, $62-67$.

19. Yang, H.L.; Zhang, C.L.; Guo, Z.L.; Liu, Y.L.; Liao, X. A hybrid model of single valued neutrosophic sets and rough sets: Single valued neutrosophic rough set model. Soft Comput. 2017, 21, 6253-6267. [CrossRef]

20. Qian, Y.H.; Liang, J.Y.; Yao, Y.Y.; Dang, C.Y. MGRS: A multi-granulation rough set. Inf. Sci. 2010, 180, $949-970$. [CrossRef]

21. Kumar, S.S.; Inbarani, H.H. Optimistic multi-granulation rough set based classification for medical diagnosis. Procedia Comput. Sci. 2015, 47, 374-382. [CrossRef]

22. Majumdar, P.; Samanta, S.K. On similarity and entropy of neutrosophic sets. J. Intell. Fuzzy Syst. 2014, 26, 1245-1252.

23. Kang, Y.; Wu, S.; Li, Y.; Liu, J.; Chen, B. A variable precision grey-based multi-granulation rough set model and attribute reduction. Knowl. Based Syst. 2018, 148, 131-145. [CrossRef]

24. Sun, B.Z.; Ma, W.M.; Qian, Y.H. Multigranulation fuzzy rough set over two universes and its application to decision making. Knowl. Based Syst. 2017, 123, 61-74. [CrossRef]

25. Pan, W.; She, K.; Wei, P. Multi-granulation fuzzy preference relation rough set for ordinal decision system. Fuzzy Sets Syst. 2017, 312, 87-108. [CrossRef]

26. Huang, B.; Guo, C.; Zhuang, Y.; Li, H.; Zhou, X. Intuitionistic fuzzy multi-granulation rough sets. Inf. Sci. 2014, 277, 299-320. [CrossRef]

27. Zhang, X.; Miao, D.; Liu, C.; Le, M. Constructive methods of rough approximation operators and multi-granulation rough sets. Knowl. Based Syst. 2016, 91, 114-125. [CrossRef]

28. Bo, C.X.; Zhang, X.; Shao, S.T.; Smarandache, F. Multi-granulation neutrosophic rough sets on a single domain and dual domains with applications. Symmetry 2018. accepted. [CrossRef]

29. Yang, H.L.; Guo, Z.L.; She, Y.; Liao, X.W. On single valued neutrosophic relations. J. Intell. Fuzzy Syst. 2016, 30, 1045-1056. [CrossRef]

30. Zhang, X.H. Fuzzy anti-grouped filters and fuzzy normal filters in pseudo-BCI algebras. J. Intell. Fuzzy Syst. 2017, 33, 1767-1774. [CrossRef]

31. Zhang, X.H.; Smarandache, F.; Liang, X.L. Neutrosophic duplet semi-group and cancellable neutrosophic triplet groups. Symmetry 2017, 9. [CrossRef]

(C) 2018 by the authors. Licensee MDPI, Basel, Switzerland. This article is an open access article distributed under the terms and conditions of the Creative Commons Attribution (CC BY) license (http://creativecommons.org/licenses/by/4.0/). 